\title{
TELESSAÚDE EM TEMPOS DE COVID-19: ACOLHIMENTO, ORGANIZAÇÃO EM REDE E INTEGRAÇÃO ENSINO-SERVIÇO
}

Larissa Sapucaia Ferreira Esteves ${ }^{1}$ Ricardo Beneti ${ }^{1}$

Daniela Garcia Damaceno ${ }^{1}$

Vanessa Aparecida Ballista ${ }^{1}$

Adriana Gomes Vitório Santos ${ }^{2}$

Danielle Araújo Borsari²

Ana Paula Brambilo Menegasso Vieira ${ }^{1}$

\author{
https://orcid.org/0000-0003-3489-2599 \\ https://orcid.org/0000-0001-7347-976X \\ https://orcid.org/0000-0001-8656-009X \\ https://orcid.org/0000-0001-7661-0428 \\ https://orcid.org/0000-0001-7253-1933 \\ https://orcid.org/0000-0003-2873-2214 \\ https://orcid.org/0000-0002-2896-9783
}

Objetivo: relatar a experiência da articulação ensino-serviço para a implantação de Serviço de Telessaúde como mecanismos de organização do fluxo de atendimento na Rede de Atenção à Saúde em um município de médio porte no oeste paulista. Método: estudo quantitativo, descritivo sob a forma de relato de experiência. Os dados foram analisados por meio da estatística descritiva. Resultados: a implantação da estratégia foi realizada por meio da articulação entre uma universidade privada e a secretaria de saúde local, compondo o Plano de Contingência para Enfrentamento da COVID-19. Participaram da ação docentes e estudantes dos cursos de enfermagem e medicina, organizados em turnos de 12 horas, todos os dias da semana. Após 60 dias da implantação, o serviço atendeu 1.575 pessoas, sendo 921 (58,4\%) ligações, 614 (39\%) contatos via aplicativo e 40 (2,6\%) acessos via e-mail. As principais condutas foram orientações gerais (n.619/67,2\%), medidas de isolamento domiciliar (n.150/16,3\%), encaminhados ao Centro de Triagem ou Unidade de Saúde (n.95/10,3\%), encaminhamento à Unidade de Pronto Atendimento (n.57/6,2\%). Conclusão: a ação se mostrou robusta como estratégia de enfrentamento da atual pandemia, principalmente em relação ao acolhimento das necessidades singulares dos usuários, bem como na colaboração com a organização da rede de atenção à saúde. Descritores: Pandemias; Infecções por Coronavirus; Telemedicina; Assistência à Saúde; serviços de Integração Docente-Assistencial.

\section{TELEHEALTH IN COVID-19 TIMES: USER EMBRACEMENT, NETWORK ORGANIZATION AND TEACHING-SERVICE INTEGRATION}

Objetive: to report the experience of the teaching-service articulation for the implementation of the Telehealth Service as mechanisms for organizing the flow of care in the Health Care Network in a medium-sized municipality in western São Paulo. Method: quantitative, descriptive study in the form of an experience report. The data were analyzed using descriptive statistics. Results: the implementation of the strategy was carried out through the articulation between a private university and the local health department, composing the Contingency Plan for Coping with COVID-19. Teachers and students of nursing and medicine courses participated in the action, organized in 12 -hour shifts, every day of the week. After 60 days of implementation, the service reached 1,575 people, with 921 (58.4\%) calls, 614 (39\%) contacts via application and 40 (2.6\%) accesses via email. The main conducts were general guidelines (n.619 / 67.2\%), home isolation measures (n.150 / $16.3 \%$ ), sent to the Screening Center or Health Unit (n.95 / 10.3\%), referral to the Emergency Care Unit (n.57 / 6.2\%). Conclusion: the action proved to be robust as a strategy to face the current pandemic, mainly in relation to welcoming the unique needs of users, as well as in collaboration with the organization of the health care network.

Descriptors: Pandemics; Coronavirus Infecions; Telemedicina; Delivery of Health Care; Teaching Care Integration Services.

\section{TELESALUD EN TIEMPOS DE COVID-19: ACOGIMIENTO, ORGANIZACIÓN DE RED E INTEGRACIÓN ENSEÑANZA-SERVICIO}

Objetivo: informar la experiencia de la articulación del servicio de enseñanza para la implementación del Servicio de telesalud como mecanismos para organizar el flujo de atención en la Red de Atención de Salud en un municipio de tamaño medio en el oeste de São Paulo. Método: estudio cuantitativo, descriptivo en forma de informe de experiencia. Los datos se analizaron mediante estadística descriptiva. Resultados: la implementación de la estrategia se llevó a cabo mediante la articulación entre una universidad privada y el departamento de salud local, componiendo el Plan de contingencia para hacer frente a COVID-19. Profesores y estudiantes de cursos de enfermería y medicina participaron en la acción, organizada en turnos de 12 horas, todos los días de la semana. Después de 60 días de implementación, el servicio llegó a 1,575 personas, con 921 (58.4\%) llamadas, 614 (39\%) contactos a través de la aplicación y 40 (2.6\%) accesos por correo electrónico. Las principales conductas fueron pautas generales (n. 619 / 67.2\%), medidas de aislamiento en el hogar (n. 150 / 16.3\%), enviadas al Centro de Detección o Unidad de Salud (n. 95 / 10.3\% ), derivación a la Unidad de Atención de Emergencia (n. 57 / 6.2\%). Conclusión: la acción demostró ser robusta como estrategia para enfrentar la pandemia actual, principalmente en relación con la satisfacción de las necesidades únicas de los usuarios, así como en colaboración con la organización de la red de atención médica.

Descriptores: Pandemias; Infecciones por Coronavirus; Telemedicina; Prestación de Atención de Salud; Servicios de Integración Docente-Asistencial.

${ }^{1}$ Universidade do Oeste Paulista - UNOESTE, Presidente Prudente, SP. 'Secretaria Municipal de Saúde de Presidente Prudente, SP. Autor Correspondente: Larissa Sapucaia Ferreira Esteves E-mail: larissaesteves@unoeste.br Recebido: 25/5/2020 Aceito: 09/6/2020 


\section{INTRODUÇÃO}

O surgimento e propagação da COVID-19, a doença causada pelo novo coronavírus (SARS-CoV-2), no final de 2019 , tomou rápida proporção, tendo sido declarada em 30 de janeiro de 2020 pela Organização Mundial da Saúde (OMS) como uma Emergência de Saúde Pública de Interesse Internacional e em 11 de março caracterizada como pandemial. Até o dia 25 de maio de 2020 contabilizavam-se 5.424 .718 pessoas contaminadas em todo o mundo, com 345.296 mortes. No Brasil, este número perfazia 363.211 confirmados e 22.666 óbitos, sendo o segundo país em número de casos e sexto colocado em mortes pela doença, variando conforme faixas etárias e condições clínicas associadas²

Os primeiros casos de pneumonia pela Covid-19 foram diagnosticados em dezembro de 2019, na China em pacientes com ligação a mercados atacadistas de frutos do mar e animais silvestres. O patógeno foi identificado como um betacoronavirus, sendo nomeado como síndrome respiratória aguda grave causada pelo coronavírus ${ }^{2,1}$.

Os betacoronavírus são um grande grupo de ácido ribonucleico - RNA-vírus que causam infecções respiratórias e intestinais com sintomatologia leve em humanos por serem ocasionadas por espécies de baixa patogenicidade 3 . Duas espécies altamente patogênicas foram responsáveis por surtos que representaram grande ameaça à saúde pública nos anos de 2002 e 2012 na China e alguns países do Oriente Médio. O SARS-CoV, vírus transmitidos por gatos, levou a óbitos milhares de pessoas por Síndrome Respiratória Aguda Severa em um surto em 2002 na China. Em 2012, o MERS-Cov também ocasionou inúmeras mortes pela chamada Síndrome Respiratória do Oriente Médio, que era transmitidas por camelos. Logo, sabe-se que o SARS-CoV-2 é de origem zoonótica, contudo seu reservatório animal não é completamente conhecido 4 .

Sua transmissão inicial na China se deu por meio de um fenômeno denominado "spillover", no qual seres humanos se contaminam pelo consumo e manuseio de produtos de origem animal infectados. A transmissão entre seres humanos pode se dar de diferentes formas ${ }^{5}$, entretanto, como a maioria das doenças respiratórias, as principais formas de contágio descritas na literatura são as gotículas e o contato com materiais contaminados ${ }^{3}$. Contudo, estudos preliminares indicam a presença do vírus inclusive em secreções oculares e lágrimas de pacientes contaminados ${ }^{6}$. Devido à falta de conhecimentos acerca de todo seu espectro de transmissão, considera-se, ainda, que o contato desprotegido com sangue, fezes, vômitos e urina implica, também, no risco de contaminação ${ }^{7-8}$

Os sintomas da Covid-19 aparecem após um período de incubação de aproximadamente 5,2 dias. Sabe-se que o vírus tem alta transmissibilidade, sendo a sua capacidade de contágio de aproximadamente 2,74 pessoas a partir do caso índice? Os sintomas mais frequentes são febre, tosse e fadiga, e em casos graves dispneia. Outros sinais e sintomas, como dor de cabeça, mialgia, hemoptise, linfopenia, e sintomas gastrointestinais podem estar presentes. Em idosos a doença pode apresentar sinais de alerta incomuns e variados, dentre eles confusão mental aguda, agitação, sonolência, prostração, ausência de febre, dificuldade em deambular, quedas, ingesta alimentar diminuida, disfagia, incontinência, mialgia e/ou mal estar geral ${ }^{10}$

Contudo, além de sua alta transmissibilidade e seus sintomas inespecíficos, restam ainda muitos detalhes a serem esclarecidos, dificultando a identificação dos sinais e sintomas da doença de fato, gerando dúvidas e incertezas para os profissionais de saúde e insegurança para a população.

A Atenção Primária à Saúde (APS) é a porta de entrada do Sistema Único de Saúde (SUS). Durante surtos e epidemias, tem papel fundamental na resposta global à doença em questão, oferecendo atendimento resolutivo e mantendo a longitudinalidade e a coordenação da rede de cuidado em todos os níveis de atenção. É a APS que tem grande potencial de identificar precocemente casos graves a serem manejados em serviços especializados. Para tanto, a APS utiliza-se de estratégias e tecnologias de saúde de diferentes densidades tecnológicas para responder às necessidades frente à pandemiall.

Neste sentido, no contexto de desastres e emergências de saúde pública, estudos descrevem as Tecnologias da Informação e Comunicação (TIC) como potentes aliadas da APS no direcionamento e acesso da população a serviços de saúde nos diferentes pontos da rede de cuidado ${ }^{12}$. Essas ferramentas são fundamentais para a redução da variabilidade de diagnósticos, bem como para melhorar o gerenciamento clínico e prestação dos serviços de saúde em todo o mundo, facilitando o acesso e aumentando a sua qualidade, eficiência e relação custo-benefício. Assim, ajudam comunidades, superando barreiras de distância e tempo entre os profissionais de saúde e usuários, aprimorando sua comunicação ${ }^{13}$

Segundo a OMS, as evidências apontam importantes benefícios socioeconômicos para pacientes, famílias, profissionais de saúde e sistema de saúde, incluindo uma comunicação aprimorada entre prestador e usuários, além de aumentar oportunidades educacionais. Para a OMS o Telessaúde cria uma universidade sem fronteiras que promove o crescimento acadêmico e a independência, porque os participantes locais têm acesso direto a informações. É importante observar que essas parcerias oferecem benefícios mútuos ${ }^{13}$. 
Destarte, uma das estratégias centrais para o controle de surtos é a "triagem direta", ou seja, a classificação dos usuários antes que estes se desloquem aos serviços de saúde. Assim, a telemedicina direta ao consumidor (ou sob demanda), permite que os usuários sejam rastreados com eficiência, protegendo-os, profissionais de saúde e comunidade da exposição ao patógeno, evitando a superlotação dos serviços de saúde ${ }^{14}$

Frente a este contexto, criou-se, então, um serviço de teleatendimento como solução sustentável para a organização do fluxo de atendimento da população na rede de atenção à saúde de um município de médio porte do oeste estado de São Paulo. Pactuado a partir da articulação de uma universidade e secretaria municipal de saúde e compondo o Plano de Contingência local. Foi desenvolvido com o intuito de colaborar com a organização e direcionamento do acesso da população ao dispositivo de atenção a saúde adequado para cada situação clínica, neste momento de crise.

Dessa forma, o objetivo desse trabalho é relatar a experiência da articulação interprofissional e de ensino-serviço para a implantação do Serviço de Telessaúde, como mecanismo de organização do fluxo de atendimento na rede de atenção à saúde em um município de médio porte no oeste paulista.

\section{MÉTODO}

Trata-se de um relato de experiencia acerca da implantação, em momento de pandemia por Covid-19, do Serviço de Telessaúde em um município que possui 218.544 habitantes situado no extremo oeste do estado de São Paulo.

A Secretaria Municipal de Saúde (SMS) da referida cidade oferece atendimento à população por meio de 24 unidades de Estratégia de Saúde da Família (ESF), representando uma cobertura populacional de 37\%, com 11 Unidades Básicas de Saúde (UBS) distribuídas em todo território. Para atendimento de urgência e emergência conta com duas Unidades de Pronto Atendimentos (UPA Zona Norte e UPA Zona Sul) e dois Pronto Atendimentos localizados na região leste e oeste da cidade. A Rede de Atenção à Saúde local conta com um hospital público de grande porte, referência para o atendimento de alta complexidade para os 45 municipios que compõe a XI Direção Regional de Saúde do Estado, além de um hospital filantrópico de médio porte e dois hospitais particulares de pequeno porte.

A implantação da estratégia foi realizada por meio de parceria ensino-serviço entre uma universidade privada e a SMS. A operacionalização do atendimento foi oferecida por docentes dos cursos de enfermagem e medicina ao comitê gestor de crise da SMS antes mesmo dos primeiros casos da doença na cidade. Após discussão das estratégias de atendimento e pactuação do serviço, o Telessaúde passou a compor o Plano de Contingência Municipal para enfrentamento da COVID-19. No âmbito da universidade, foi oficializado como Projeto de Extensão Universitária (Protocolo n. 14951/2020) uma vez que estudantes dos cursos de enfermagem e medicina, junto aos docentes dos mesmos cursos, seriam os responsáveis pelo teleatendimento

A população alvo do projeto se constituiu dos habitantes da cidade que acessassem o serviço, bem como dos municipios vizinhos. Os atendimentos foram pactuados para ocorrerem todos os dias da semana, das $8 \mathrm{~h}$ às $20 \mathrm{~h}$.

Tanto para o serviço, como para o ensino, a estratégia teve como objetivos: acolher a população usuária do Sistema Único de Saúde, via telefone, em relação a doença COVID-19; esclarecer dúvidas quanto as características clínicas, epidemiológicas da doença e fatores de risco associados; auxiliar a tomada de decisão clínica; direcionar e organizar o fluxo de atendimento estabelecido pela SMS; vincular os usuários que apresentassem sinais e sintomas de gravidade causados pela possível infecção causada pelo coronavirus às unidades de referência; orientar as medidas de contenção estabelecidas pelo Ministério da Saúde tais como lavagem das mãos, uso do álcool em gel, isolamento social e domiciliar; orientar acerca das mudanças ocorridas nos atendimentos à saúde dos usuários portadores de doenças crônicas não transmissiveis, pessoas com mais de 60 anos, gestantes, crianças, bem como nas rotinas de consultas, exames, retiradas de medicamentos e vacinas; desenvolver no estudante compromisso, corresponsabilidade frente à situações de crise na saúde e conhecimento em relação a nova doença causada pelo coronavirus.

A pactuação do serviço ocorreu no dia 18 de março de 2020, no dia 23 estudantes e docentes receberam treinamento dos coordenadores do projeto e da SMS e no dia 24 de março iniciaram-se os atendimentos, com previsão de término junto ao fechamento do Plano de Contingência Municipal. Para apresentação dos resultados da experiencia, os dados quantitativos foram analisados por meio da estatística descritiva.

\section{DESCRIÇÃO DA EXPERIÊNCIA}

O Telessaúde foi implantado fisicamente nas dependências da universidade que disponibilizou sala ampla e arejada, com lavatório, cinco computadores, uma linha telefônica específica para atendimento à população com cinco ramais telefônicos, uma linha de telefone móvel para atendimentos via aplicativo multiplataforma de mensagens instantâneas e e-mail próprio, além de mesas, cadeiras e bloco de papel (flipchart) com cavalete. Os equipamentos foram dispostos se- 
guindo normas de distanciamento de dois metros entre eles, garantido limpeza local três vezes ao dia, com abastecimento de álcool em gel, álcool a 70\% e papel toalha para desinfeção dos equipamentos.

Os estudantes do último ano da graduação de enfermagem e medicina foram recrutados, bem como docentes enfermeiros e médicos que voluntariamente se candidataram na efetivação da prestação do serviço. Todos os voluntários receberam treinamento específico da universidade, através do seu corpo docente especializado, nos aspectos inerentes à patologia em questão, sobre peculiaridades da organização da rede de atenção do município e do Ministério da Saúde por meio do programa "O Brasil conta comigo" versão profissional e versão acadêmico. A equipe foi distribuída em turnos de 4 horas, sendo composta por dois estudantes de cada curso, sob a supervisão de um docente enfermeiro e um médico.

A iniciativa fundamentou-se em quatro princípios: fornecer suporte clínico, superar barreiras geográficas (conectando usuários que não estão no mesmo local físico garantindo orientação adequada na malha assistencial), explorar o uso de vários tipos de TIC na comunicação usuário/sistema de saúde e melhorar os resultados de saúde.

O desenvolvimento do trabalho foi orientado pelos princípios das Tecnologias em Saúde, compondo a ideia de que a dimensão relacional é central no processo de trabalho em saúde e comporta um tipo de tecnologia, pois o modo de estabelecer estas relações é tecnologicamente orientado. Assim, as 'tecnologias leves', que associam a produção de vínculo, autonomização, acolhimento e gestão e as 'tecnologias leve-duras' que se sustentam por meio dos saberes já estruturados ${ }^{15}$, serviram de base para compor a lógica do trabalho.

Para o registro dos atendimentos, a coordenação do programa, com apoio da SMS, elaborou planilhas no Programa Excel ${ }^{\circledR}$ garantindo que dados essenciais fossem coletados para subsidiar os relatórios que deveriam ser entregues. A planilha de atendimento contém os seguintes dados: nome do estudante, curso, data e hora do atendimento, nome do usuário, telefone, idade, sexo, unidade de saúde ao qual está inserido; rastreio de sintomas para COVID-19, antecedentes epidemiológicos, condições clínicas de risco, sinais e sintomas de agravamento, conduta do atendente e nome do responsável pelo recebimento da referência à UPA, nos casos específicos. Os dados obtidos se destinam a análises epidemiológicas bem como para avaliação dos resultados do projeto.

Foram elaborados dois fluxogramas, um para tomada de decisão no atendimento de caso primário e o segundo para atendimento de caso suspeito ou confirmado COVID-19 em observação domiciliar, ambos contemplando os pontos da rede de atenção essenciais, conforme Figura 1 e 2.

Figura 1 - Fluxograma de atendimento caso primário Projeto Telessaúde, Presidente Prudente, 2020.

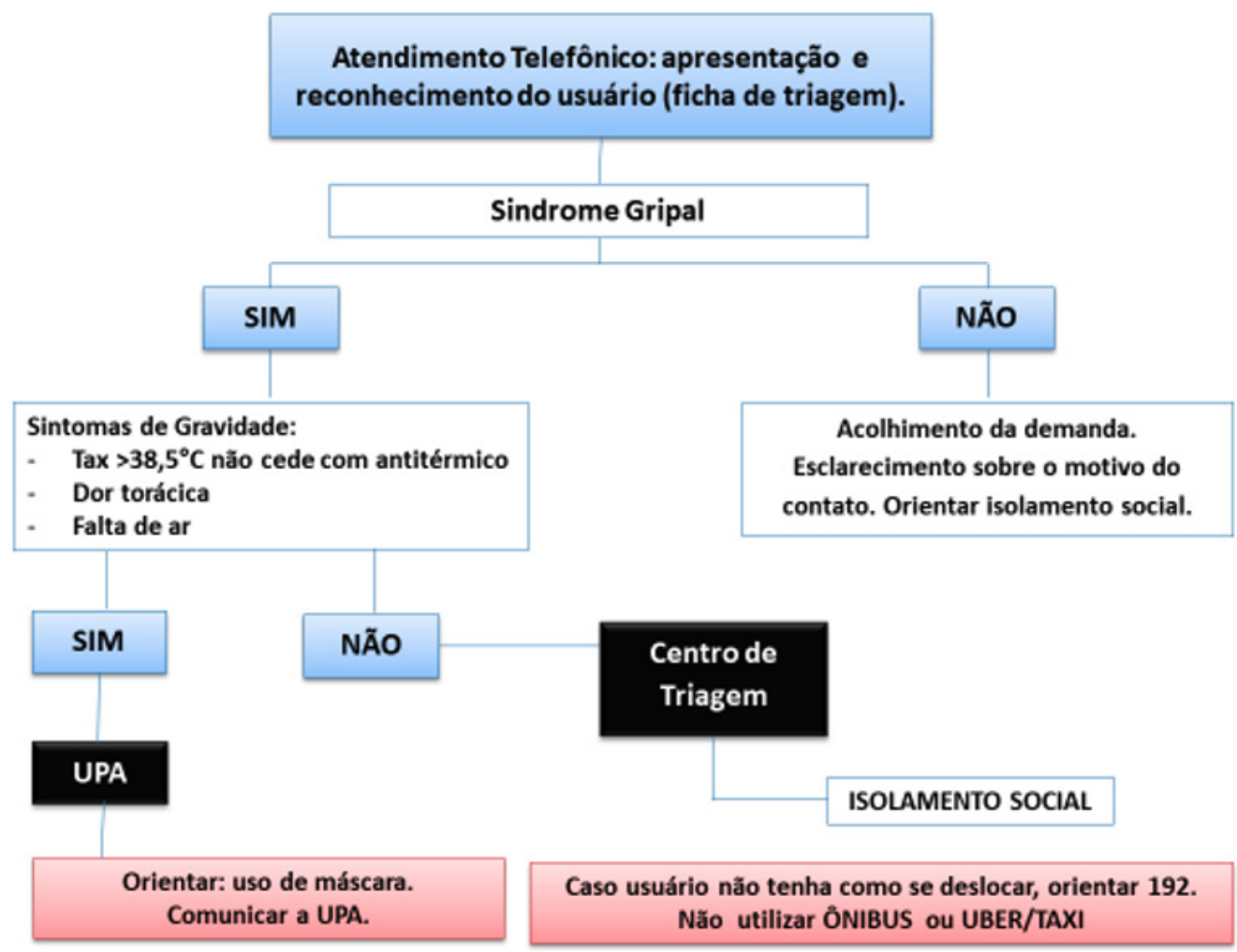


Figura 2 - Atendimento caso suspeito ou confirmado COVID-19 em observação domiciliar Projeto Telessaúde, Presidente Prudente, 2020

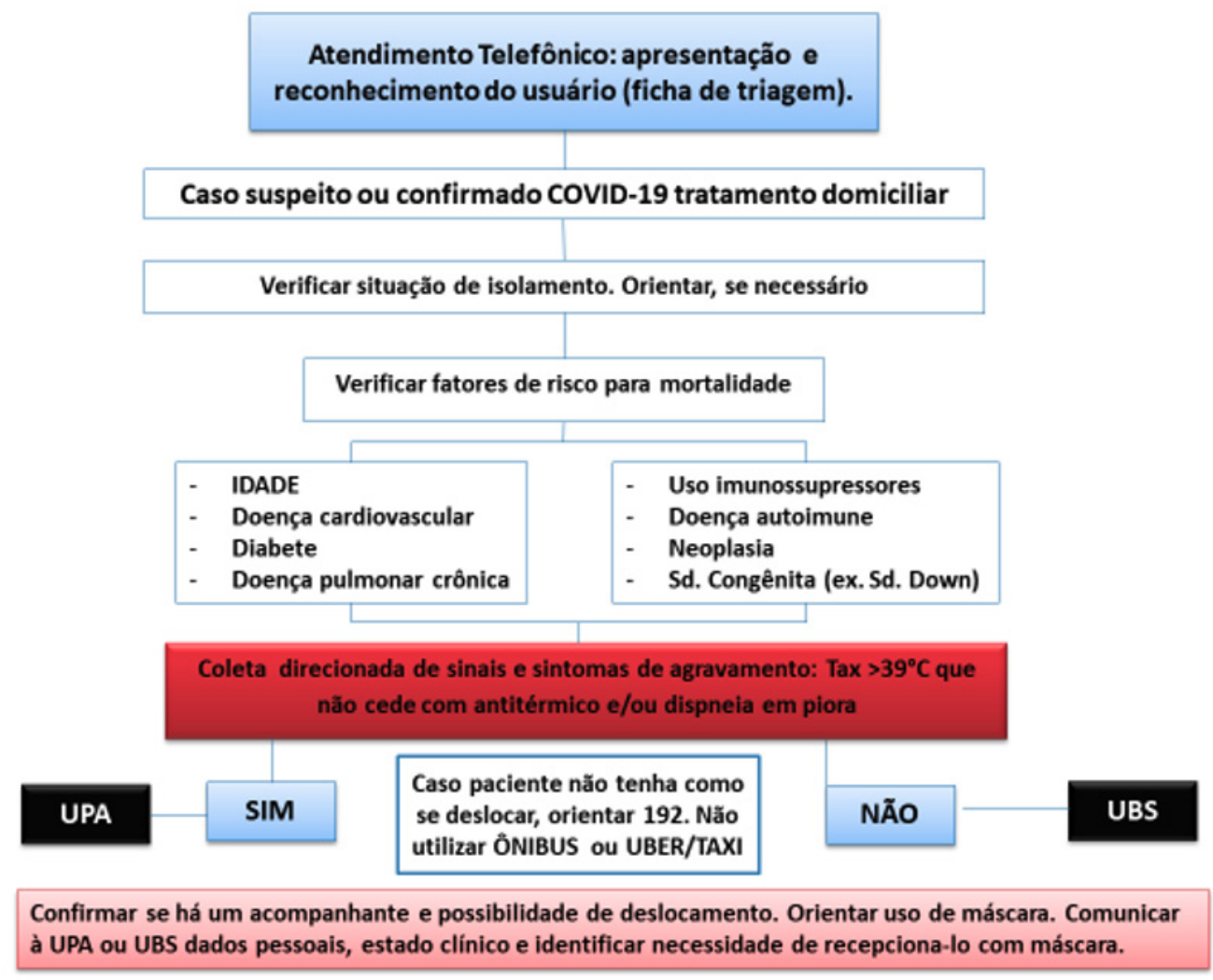

Diariamente, as mudanças ocorridas nos fluxos de atendimento, bem como informações acerca de vacinas, exames e consultas eram atualizados pela SMS. Os docentes registravam as mudanças em murais organizados nas paredes da sala, garantindo que todos os membros da equipe tivessem acesso.

Os docentes também se responsabilizaram em atualizar os estudantes por meio dos Protocolos e Diretrizes do Ministério da Saúde, sendo os principais: Protocolo de manejo clínico do coronavírus (COVID-19) na Atenção Primária à Saúde, Protocolo de Manejo Clínico da COVID-19 na Atenção Especializada, Fast-track de Teleatendimento para a Atenção Primária fluxo rápido, Campanha de vacinação contra a influenza e o sarampo na atenção primária à saúde durante a pandemia do novo coronavírus, Orientações sobre o isolamento domiciliar, SARS-CoV-2 antibody test e Teste Rápido Imunocromatográfico também compuseram os objetivos de orientações.

Para a divulgação do serviço de utilidade pública, o Telessaúde contou com o apoio das rádios locais, e jornais impressos e televisivos que realizaram reportagens explicando o objetivo da estratégia e apresentando dados que eram atualizados semanalmente pela coordenação. Contou, ainda, com a divulgação pelas redes sociais da Universidade e da SMS, além do apoio da própria população por meio do compartilha- mento dos números de telefone, aplicativos de multiplataforma de mensagens instantâneas.

\section{RESULTADOS ALCANÇADOS}

Em 60 dias de operacionalização do Telessaúde, a equipe realizou 1.575 atendimentos, sendo 921 (58,4\%) ligações, 614 (39\%) contatos via aplicativo multiplataforma de mensagens instantâneas e 40 (2,6\%) acessos via e-mail. A caracterização dos contatos realizados via telefone mostra que a maioria foram realizados por mulheres (n.628/68,2\%), que estavam em busca de informações e orientações para tomada de decisão junto aos seus familiares. A média de idade das pessoas que procuraram pelo serviço foi de 53,42 anos (máx. 93/min.17/dp.15,26 anos)

Observou-se que o aplicativo WhatsApp, mostrou-se uma ferramenta potente no gerenciamento dos casos, devido a possibilidade de melhor exploração dos sintomas e detalhes clínicos, assim como a utilização de suas funcionalidades como envio de documentos, fotos e vídeos.

Ressalta-se que, embora o Telessaúde tenha sido desenvolvido com foco no manejo e educação em saúde acerca da pandemia do coronavírus, durante o "trabalho vivo em ato"15 mostrou-se sua potencialidade no manejo de outras situações clínicas. Nesse sentido, para além dos resultados espe- 
ARTIGO 27

rados observou-se um impacto importante no manejo do paciente com suspeita e confirmação de dengue e transtornos de ansiedade relacionados ao isolamento social.

Quanto as condutas tomadas pela equipe de saúde do Telessaúde, 619 (67,2\%) das ligações demandaram orientações sobre vacina, consultas, exames, receitas e novas rotinas de atendimento da Atenção Primária de Saúde do município, $150(16,3 \%)$ pessoas foram orientadas quanto a medidas de isolamento domiciliar, 95 (10,3\%) foram encaminhados ao Centro de Triagem para COVID-19 ou Unidade de Saúde, 57 (6,2\%) apresentaram sinais que justificaram o encaminhamento à Unidade de Pronto Atendimento.

É valido ressaltar que as orientações quanto ao isolamento social e encaminhamentos eram tomadas em discussões entre os discentes e docentes seguindo as orientações dos fluxogramas de atendimento aos pacientes com síndrome gripal orientados pelo Ministério da saúde.

Esta experiencia proporcionou, ainda, aos discentes de enfermagem e medicina, a partir da discussão dos casos o desenvolvimento da capacidade de análise e síntese, gestão da informação, tomada de decisões e resoluções de problemas.

Evidenciou-se, então, que a forma com a qual foi o serviço foi construído, pautado na articulação de diferentes Tecnologias de Informação e Comunicação e Tecnologias de Saúde, possibilitou o desenvolvimento de um trabalho problematizador, reflexivo e resolutivo, com impactos positivos para a assistência a saúde da população e para a formação em saúde.

\section{Limitações do estudo}

Trata-se de uma experiência vivenciada em um município de médio porte no extremo oeste paulista, em que os dados e resultados podem não expressar a realidade das cidades que compõe o Brasil continental. Considera-se importante reforçar que a estratégia foi implantada antes mesmo da confirmação dos primeiros casos da doença na região, oferecendo um canal de comunicação para esclarecimentos e dúvidas apresentadas pela população na vigência de uma ameaça real. Esse fato justifica o expressivo número de atendimentos para situações não relacionadas aos sinais e sintomas da doença.

\section{Contribuições para a prática}

O Telessaúde mostrou-se uma estratégia robusta no que tange o acolhimento e a organização do fluxo de atendimento da rede de atenção à saúde local, contribuindo principalmente como um canal de comunicação simples e de fácil acesso, atendendo o usuário em suas demandas considerando especialmente, a singularidade da necessidade de cada pessoa.
A integração ensino-serviço-comunidade representou relevante estratégia para a efetivação do SUS ao articular e buscar sinergia entre a formação profissional, oferecida pelos cursos de graduação, e o serviço de atenção à saúde ofertado no SUS. A necessidade de integração dos saberes profissionais (doentes, estudantes e profissionais dos serviços de saúde) se fizeram fundamentais para a construção diária de consensos frente a avalanche de informações produzidas em situações de pandemia e assim, fornecer informações consistentes aos usuários.

\section{CONSIDERAÇÕES FINAIS}

É possivel afirmar que a articulação entre a universidade e o secretaria municipal de saúde do município estudado repercutiu positivamente na construção do trabalho coletivo e interdisciplinar, construiu possibilidades de atuação resolutiva e satisfatória, que possibilitam o fortalecimento dos princípios da longitudinalidade e integralidade do cuidado, reforçando a importância dessa articulação para o Sistema Único de Saúde.

Identifica-se que o Telessaúde mostrou-se como uma ferramenta importante no contexto da pandemia, diminuindo os obstáculos de acesso da população a informações e a serviços de saúde. Assim, ressalta-se, também, seu impacto fundamental na redução da transmissão da doença com o controle do trânsito desnecessário de pessoas com sintomatologia leve em serviços de saúde.

Por fim, denota-se a importância da presença dos estudantes de saúde nesse espaço potente para a sua formação. Essas experiências contribuem para mudanças nas práticas desses futuros profissionais, por meio da aprendizagem de forma crítica e reflexiva, impactando diretamente em uma atenção mais ampliada ao usuário e valorização do SUS.

Contribuição dos Autores: 1, 2 e 3: concepção e/ou desenho do estudo; coleta, análise e interpretação dos dados; redação e/ou revisão crítica do manuscrito; aprovação da versão final a ser publicada;4, 5, 6 e 7: articulação para implantação da experiência, coleta de dados; revisão crítica do manuscrito; aprovação da versão final a ser publicada.

Agradecimentos: os autores agradecem as autoridades que auxiliaram o projeto, disponibilizando a estrutura física e o suporte institucional para sua concretização. A secretaria Municipal de Saúde de Presidente Prudente que confiaram e acreditaram que na potência da parceria ensino-serviço para o atendimento à comunidade. Aos inúmeros voluntários, docentes e discentes da Unoeste e demais profissionais de saúde nos fazem acreditar que vale sempre a pena investir na humanidade. 


\section{REFERÊNCIAS}

1. W. Guan, Ni Z, Hu Y, Liang W, Ou C, He J et al. Clinical Characteristics of Coronavirus Disease 2019 in China. The new england journal of medicine [internet]. Feb 2020. [cited 2020 Mai 05] Available Doi: https:// doi.org/10.1056/NEJMoa2002032

2. Johns Hopkins University (JHU). COVID-19 Dashboard by the Center for Systems Science and Engineering (CSSE) at Johns Hopkins University [internet]. 2020 [cited 2020 Mai 25] Available from https:// coronavirus.jhu.edu/

3. World Health Organization (WHO)a. Novel Coronavirus (2019-nCoV) technical guidance [internet]. 2020 [cited 2020 Mai 15]. Available from: https://www.who.int/emergencies/diseases/novel-coronavirus-2019.

4. Rothan HA, Byrareddy SN. The epidemiology and pathogenesis of coronavirus disease (COVID-19) outbreak. Journal of Autoimmunity [internet]. 2020 [cited 2020 Mai 15]; v. 109, May 2020, 102433. Available from Doi: https://doi.org/10.1016/j.jaut.2020.102433

5.Ministério da Saúde (BR). COVID-19. Painel Coronavírus [internet]. Brasília, 2020 [cited 2020 Mai 25]. Available from: https://covid.saude.gov.br/

6. Xia J, Tong J, Liu M, Shen Y, Guo D. Evaluation of coronavirus in tears and conjunctival secretions of patients with SARS CoV 2 infection. J Med Virology [internet]. 2020 [cited 2020 Mai 05]; 92: 589-594. Available from Doi: https://doi.org/10.1002/jmv.25725

7. Center for Disease Control and Prevention (CDC). Interim U.S. guidance for risk assessment and public health management of healthcare personnel with potential exposure in a healthcare setting to patients with Coronavirus Disease (COVID-19) [internet]. 2020 [cited 2020 Mai 15]. Available from: https://www.cdc. gov/coronavirus/2019-ncov/hcp/guidance-risk-assesment-hcp.html

8. Del Rio C, Malani PN. Novel Coronavirus-Important Information for Clinicians. JAMA [Internet]. 2020 [cited 2020 Mai 15]. 323(11):1039-40. Available from DOI: https://doi.org/10.1001/jama.2020.1490.

9. Li Q, Guan X, Wu P, Wang X, Zhou L, Yeqing Tong Y, et al. Early transmission dynamics in Wuhan, China, of novel coronavirus-infected pneumonia. N. Engl. J. Med [internet]. 2020 [cited 2020 Mai 18]; 382:1199-1207. Available from https://doi.org/10.1056/NEJMoa2001316

10. Li T, Lu H, Zhang W. Clinical observation and management of COVID-19 patients. Emerging Microbes 8 Infections [internet]. 2020 [cited 2020 Mai 18]; 9 (1), 687-690. Available from https://doi.org/10.1080/222 21751.2020 .1741327

11. Ministério da Saúde (BR). Protocolo de Manejo Clínico do Coronavírus (Covid-19) na Atenção Primária à Saúde [internet]. Versão 7. Brasília, 2020 [cited 2020 Mai 18]. Available from: https://www.unasus.gov.br/ especial/covid19/pdf/37

12. Lurie N, Carr BG. The role of telehealth in the medical response to disasters. JAMA Intern Med [internet] 2018 [cited 2020 Mai 05]; 178:745-746. Available from: https://jamanetwork.com/journals/jamainternalmedicine/article-abstract/2678828

13. World Health Organization (WHO). Telemedicine: opportunities and developments in member states. Report on the second global survey on Health [internet]. World Health Organization, 2010 [cited 2020 Mai 05]. Available from: https://apps.who.int/iris/bitstream/handle/10665/44497/9789241564144_eng.pdf?sequence $=1$ EisAllowed $=y$

14. Hollander JE, Carr BG. Virtually perfect? Telemedicine for Covid-19. N Engl J Med [internet]. 2020 [cited 2020 Mai 15]; 382:1679-1681. Available from DOI: 10.1056/NEJMp2003539

15. Franco TB, Merhy EE. Cartografias do Trabalho e Cuidado em Saúde. Tempus Actas de Saúde Coletiva [internet], 2012 [cited 2020 Mai 05]; v. 6. n. 2, 151-163. Available from DOI: http://dx.doi.org/10.18569/tempus.v6i2.1120 